\title{
Successful ATRP Syntheses of Amphiphilic Block Copolymers Poly(styrene-block- $N, N$-dimethylacrylamide) and Their Self-assembly
}

\author{
Man HuA ${ }^{1}$, Tatsuo KAneko ${ }^{2}$, Xiao-ya LiU ${ }^{1}$, Ming-qing $\mathrm{CHEN}^{1, \dagger}$, and Mitsuru AKASHI ${ }^{1,2, \dagger}$ \\ ${ }^{1}$ School of Chemical and Material Engineering, Southern Yangtze University, Wuxi 214036, P. R. China \\ ${ }^{2}$ Department of Molecular Chemistry, Graduate School of Engineering, Osaka University, \\ 2-1 Yamadaoka, Suita 565-0871, Japan
}

(Received August 25, 2004; Accepted October 28, 2004; Published February 15, 2005)

\begin{abstract}
Amphiphilic AB-type block copolymers of poly(styrene-block- $N, N$-dimethylacrylamide) (PSt- $b$ PDMAA) were successfully synthesized by atom transfer radical polymerization (ATRP) of DMAA initiated by bromide end-capped PSt (polydispersity $=1.08$ ), PSt-Br, under an appropriate catalyst/ligand system of $\mathrm{CuCl} /$ $N, N, N^{\prime}, N^{\prime}, N^{\prime \prime}$-pentamethyldiethylenetriamine, where PSt-Br was pre-synthesized by ATRP of St using ethyl- $\alpha$-bromopropionate as an initiator under $\mathrm{CuCl} / 2,2$-bipyridine. We investigated the self-assembly behavior of PSt- $b$-PDMAA with different polymerization degree (DP) of hydrophilic PDMAA block and a constant DP of PSt block in various aqueous milieus. The dynamic laser light scattering (DLLS) studies demonstrated that every PSt- $b$-PDMAA formed the self-assembled matters whose radii depended on both polymer and salt concentrations. Transmission electron microscope (TEM) and the chain length of PSt- $b$-PDMAA suggested that the present polymers formed vesicular assembly with a size-controllable hollow structure. [DOI 10.1295/polymj.37.59]

KEY WORDS Atom Transfer Radical Polymerization / Amphiphilic Block Copolymer / Polymer Vesicle / Self-assembly / Hollow Particles /
\end{abstract}

Since nanoparticles such as hollow particles have a great potential for encapsulation of large quantities of guest molecules and have a high dispersion-stability, they may have promising applications in fields of drug delivery, ${ }^{1,2}$ diagnosis, ${ }^{3}$ and separation technology. ${ }^{4}$ Although hollow particles were often prepared by the polymer deposition onto a degradable particle substrate and following removal of the substrate, ${ }^{5,6}$ these two-step methods are so complicated that one-step self-assembly method may be of scientific and industrial importance. The self-assembly of the polymer chains efficiently formed soft nanomatters based on multiple intermolecular interactions of the long chain, giving a higher stability than the case of low-mass molecule assembly. The polymer vesicles with a hollow structure have been self-assembled using block copolymers with an amphiphilic structure. ${ }^{7-10}$ Our particular interests have concentrated on the amphipathic structure where polystyrene (PSt) block as a hydrophobic segment and polyacrylamide derivatives as a hydrophilic segment, since PSt is one of the most hydrophobic polymers having a strong $\pi$-stacking interaction while the latter polymers are one of a widely-used water-soluble polymers which have high advantages for developing the biomedical materials. Especially, polydimethylacrylamide (PDMAA) is sensitive to a change in aqueous milieu due to the secondary amino side group. If PSt- $b$-PDMAA forms the vesicle, its size is controllable by environmental change. However, as far as we know, PSt- $b$-PDMAA diblock copolymers with controlled structures have not been synthesized.

The controlled living radical polymerization has attracted researchers' attention due to the reaction simplicity, facility in actual processes, and the wide controllability of the molecular structures. ${ }^{11-13}$ Especially atom transfer radical polymerization (ATRP) ${ }^{14-17}$ or reversible addition-fragmentation transfer polymerization (RAFT) $)^{18,19}$ has been successfully used to buildup the strictly-controlled architectures of block copolymers and hyper-branched polymers, ${ }^{20}$ due to the high validity and the wide adoption of various monomers. The opportunities to synthesis amphiphilic ABtype diblock copolymers have been considerably widened using the ATRP technique. Several experimental studies on homopolymerization of acrylamide-based monomers by ATRP have been so far made. ${ }^{21,22}$ On the other hand, except for a study of block copolymerization of DMAA on the surface of latexes, ${ }^{23}$ the structural control of acrylamide-based amphiphilic block copolymer by ATRP was difficult for three main reasons according to the reports; ${ }^{21}$ (1) catalyst inactivation by complexation with the polymers, (2) too strong bond between bromine and the terminal monomer unit of the polymers, and (3) nucleophilic displacement of terminal bromine by the penultimate

${ }^{\dagger}$ To whom correspondence should be addressed (E-mail: akashi@chem.eng.osaka-u.ac.jp, mqchen@sytu.edu.cn). 
amide group. Most studies on the ATRP of acrylamides have only focused on the controlled polymerization of acrylamides but the vesicular self-assembling behavior has not been systematically studied. However, $\quad N, N, N^{\prime}, N^{\prime}, N^{\prime \prime}$-pentamethyldiethylenetriamine (PMDETA) was an appropriate special ligand which can give a precise synthesis of PDMAA homopolymers according to the literature. ${ }^{21}$

In the present work, we attempt to synthesis amphiphilic block copolymers of PSt- $b$-PDMAA by ATRP using $\mathrm{CuCl} / \mathrm{PMDETA}$ system and investigate their self-assembly in water. We have established the successful ATRP method of PSt- $b$-PDMAA which showed a self-assembly to form the vesicular structure with a controllable size.

\section{EXPERIMENTAL}

\section{Materials}

Ethyl- $\alpha$-bromopropionate (EPN-Br), 2,2-bipyridine (bpy), $N, N, N^{\prime}, N^{\prime}, N^{\prime \prime}$ - pentamethyldiethylenetriamine (PMDETA), petroleum ether, and methanol were all used as received. $\mathrm{CuCl}$ (99.95\%) was purified by recrystallization. Tetrahydrofuran (THF) was purified by distillation from calcium hydride followed by distillation from sodium/benzophenone ketyl. Styrene (St) and $N, N$-dimethylacrylamide (DMAA) were purified by vacuum distillation to remove stabilizer. Water was purified by the Millipore Milli-Q system.

\section{Synthesis of PSt End-caped with Bromide (PSt-Br)}

PSt end-capped with bromide was synthesized by ATRP method by the following procedure. The ligand 2,2-bipyridine $(0.01 \mathrm{~mol})$ and the monomer St $(0.05$ mol) were added to a $100 \mathrm{~mL}$ three-neck round-bottom flask equipped with a magnetic bar and condenser which was pre-flushed with nitrogen. After the solution in the flask had been degassed with nitrogen for $20 \mathrm{~min}$, the initiator EPN-Br $(0.0005 \mathrm{~mol})$ was added via syringe, followed by addition of the catalyst $\mathrm{CuCl}$ $(0.0005 \mathrm{~mol})$. Then the flask was heated in an oil bath and stirred at $110^{\circ} \mathrm{C}$. After degassing with nitrogen for additional $20 \mathrm{~min}$, the flask was sealed with rubber septum and the reaction solution was stirred for a given period. The reaction proceeded homogeneously and the solution became somewhat viscous. After the solution was cooled to room temperature spontaneously, it was dissolved in THF $(20 \mathrm{~mL})$ and then was reprecipitated in the methanol $(150 \mathrm{~mL})$. The reprecipitation was repeated twice. The precipitates were dried in vacuo overnight, to produce the PSt end-caped with bromide (PSt-Br).

\section{Synthesis of PSt-b-PDMAA Copolymers}

PSt- $b$-PDMAA was synthesized by ATRP method by the following procedure. The ligand PMDETA $(0.0005 \mathrm{~mol})$, the monomer DMAA $(0.0375 \mathrm{~mol})$ and the macroinitiator PSt-Br $(0.00025 \mathrm{~mol})$ were added to a $100 \mathrm{~mL}$ three-neck round-bottom flask equipped with a magnetic bar and condenser which was preflushed with nitrogen. The catalyst $\mathrm{CuCl}(0.0005$ mol) was added after the mixture had been degassed with nitrogen for $20 \mathrm{~min}$, and then the reaction mixture was stirred at $110^{\circ} \mathrm{C}$. After degassing with nitrogen for additional $20 \mathrm{~min}$, the flask was sealed with rubber septum. The reaction mixture was stirred for different periods $12-60 \mathrm{~h}$. The reaction proceeded homogeneously and the solution became more viscous than the mixture in the initial state. After the mixture was cooled to room temperature spontaneously, it was dissolved in THF $(20 \mathrm{~mL})$ and filtered through a basic alumina plug to remove catalyst. The products of PSt$b$-PDMAA were precipitated in the petroleum ether (repeated twice) and dried in vacuo overnight.

\section{Self-assembling of PSt-b-PDMAA Copolymers}

The method reported by Eisenberg ${ }^{24}$ was used to prepare micelles in the present study. The block copolymers PSt- $b$-PDMAA ( $c a$. $0.01 \mathrm{~g}$ ) were first dissolved in dimethylformamide (DMF, $10 \mathrm{~mL}$ ) and the solutions were filtered. Subsequently, the solutions of the block copolymer were added very slowly to the mildly stirred water until the solution turned cloudy and had bluish turbidity, resulting in polymer concentrations of $8 \times 10^{-5}-4 \times 10^{-4} \mathrm{~g} \mathrm{~mL}^{-1}$. The resulting solutions were then dialyzed against distilled water for $3 \mathrm{~d}$ to remove DMF. Porous membranes (UC3632-100) were used for dialysis. The dialyzed solutions were used as DLLS and TEM measurement.

\section{Characterizations}

Molecular weights and molecular weight distribution were measured on a gel permeation chromatography (GPC; Polymer Standards Service columns) system consisting of Waters 515 pump and a Waters 410 refractive-index detector against polystyrene standards, using DMF as an eluent.

The proton nuclear magnetic resonance $\left({ }^{1} \mathrm{H}\right.$ NMR) spectra (Bruker DMX500) were obtained at $500 \mathrm{MHz}$ in $\mathrm{CDCl}_{3}$ at room temperature. ${ }^{1} \mathrm{H} \mathrm{NMR}$ chemical shifts in parts per million ( $\mathrm{ppm}$ ) were recorded downfield from $0.00 \mathrm{ppm}$ using tetramethylsilane (TMS) as an internal reference.

Fourier transformation infrared (FT-IR) spectra were recorded on FTLA2000-104 (Canada Bomem Co.) spectrometers after 64 scans $\left(4 \mathrm{~cm}^{-1}\right.$ resolution) over the range from 4000 to $400 \mathrm{~cm}^{-1}$. The samples were mixed with $\mathrm{KBr}$ by grinding and pressed to form the thin pellet as a specimen.

A dynamic laser light scattering spectrometer 


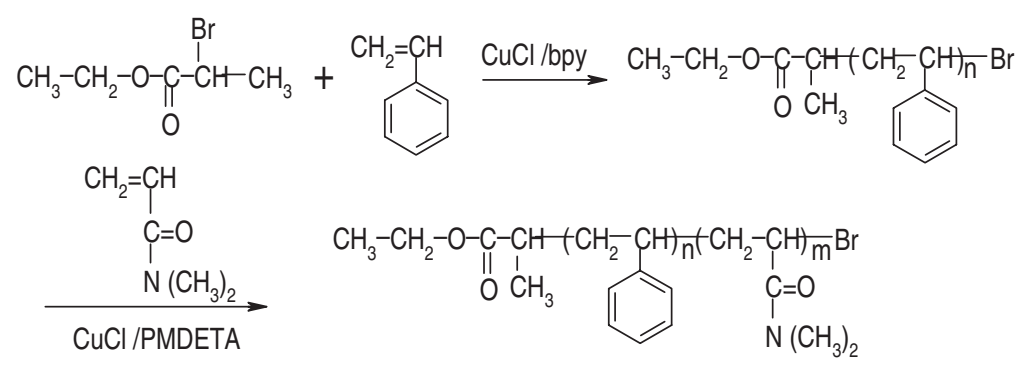

Scheme 1. The synthetic root and the structure of PSt- $b$-PDMAA by ATRP.

Table I. The characterization of PSt- $b$-PDMAA copolymers synthesized by ATRP ${ }^{a}$

\begin{tabular}{lccccccc}
\hline Abb. & $\begin{array}{r}\text { R.t. } \\
(\mathrm{h})^{\mathrm{b}}\end{array}$ & $\begin{array}{c}\text { Conv. } \\
(\%)\end{array}$ & $\begin{array}{c}M_{\mathrm{n}, \mathrm{GPC}}{ }^{\mathrm{c}} \\
\left(\times 10^{-3}\right)\end{array}$ & $\begin{array}{c}M_{\mathrm{n}, \mathrm{th}}{ }^{\mathrm{c}} \\
\left(\times 10^{-3}\right)\end{array}$ & $M_{\mathrm{w}} / M_{\mathrm{n}}$ & $m / n^{\mathrm{d}}$ & $\begin{array}{c}\text { Length }^{\mathrm{e}} \\
(\text { PDMAA/PSt; nm) }\end{array}$ \\
\hline P32 & 12 & 25 & 6.0 & 6.5 & 1.41 & $32 / 25$ & $8.0 / 6.3$ \\
P77 & 24 & 50 & 10.5 & 10.2 & 1.50 & $77 / 25$ & $19.3 / 6.3$ \\
P101 & 36 & 65 & 12.8 & 12.4 & 1.55 & $101 / 25$ & $25.3 / 6.3$ \\
P121 & 48 & 78 & 14.8 & 14.4 & 1.61 & $121 / 25$ & $30.3 / 6.3$ \\
P139 & 60 & 90 & 16.6 & 16.2 & 1.61 & $139 / 25$ & $34.8 / 6.3$ \\
\hline
\end{tabular}

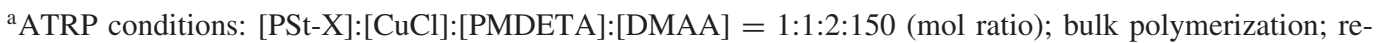
action temperature: $110^{\circ} \mathrm{C}$; Number-average molecular weight $M_{\mathrm{n}}$ of PSt-Br $=2800 .{ }^{\text {b }}$ R.t. refers to the reaction time. ${ }^{\mathrm{c}} M_{\mathrm{n}, \mathrm{GPC}}$ refers to the Mn determined by GPC methods and $M_{\mathrm{n}, \text { th }}$ refers to Mn calculated theoretically. ${ }^{\mathrm{d}} m$ is the number of PDMAA units; $n$ is the number of PSt units. ${ }^{\mathrm{e}}$ Block chain lengths calculated under the assumption that both chains adopted the fully-extended all-trans conformation.

(ALV500E, ALV Co., Germany) was performed to measure the hydrodynamic diameter of the micelles, which was equipped with an ALV-5000 multi- $\tau$ digital time correlator and a He-Ne laser (Uniphase, output power $\approx 22 \mathrm{~mW}$ at $\lambda=632.8 \mathrm{~nm}$ ). The scattering angel was $45^{\circ}$. A refractive index matching bath of filtered toluene surrounded the scattering cell, and the temperature was controlled at $25^{\circ} \mathrm{C}$.

Transmission electron microscopy (TEM) images were obtained with a Hitachi 7000A microscope operated at an acceleration voltage of $150 \mathrm{kV}$ at a magnification of 100,000 times. Specimens were prepared by slow evaporation of a drop of approximately diluted solution deposited onto a collodion-coated copper mesh grid, followed by carbon spattering or by $\mathrm{OsO}_{4}$ staining. TEM was used to observe the morphology of the micelles.

\section{RESULTS AND DISCUSSION}

\section{Characterization of PSt-b-PDMAA}

Using ATRP we synthesized the amphiphilic diblock copolymers PSt- $b$-PDMAA whose structure was as follows: $m$ refers the number of hydrophilic block DMAA, while $\mathrm{n}$ refers the number of hydrophobic block PSt as shown in Scheme 1. GPC measurements showed that the molecular weights of PSt polymerized for $2 \mathrm{~h}$ was $2800 \mathrm{~g} \mathrm{~mol}^{-1}$ and polydispersity was quite small (1.08) under PSt standards. This re- sult almost agrees with the Wang's report. ${ }^{18}$ Block copolymer compositions and polydispersities of PSt$b$-PDMAA measured by GPC are summarized in Table I. The GPC curve after purification showed no peak near the elution time corresponding to $M_{\mathrm{n}}=$ 2800 (belongs to PSt-Br macromonomer), suggesting the catalyst and the unreacted monomers can be successfully removed after the purification process. One can see that ATRP of DMAA initiated by PSt-Br smoothly proceeds to give a high conversion and a narrow molecular weight distribution under these conditions. Moreover, molecular weights were successfully controlled and the experimental data were well in agreement with the values calculated based on the assumption that one molecule of the initiators realistically yielded one living polymer chain. These results indicate that using the appropriate PMDETA ligand for $\mathrm{CuCl}$ is effective on ATRP synthesis of PDMAA block copolymers. The diblock copolymer compositions calculated by GPC results were also summarized in Table I.

To assess the living character of ATRP, we studied the kinetics of the DMAA homopolymerization. As shown in Figure 1, a molecular weight increase showed a direct proportion to the conversions and the value of the polydispersity was small $\left(M_{\mathrm{w}} / M_{\mathrm{n}}<1.7\right)$. This result suggests the minimal irreversible chain transfer reaction. As shown in Figure 2, the logarithmic plot of conversion data, $\ln \left(\left[M_{0}\right] /[M]\right),([M]$ is the monomer 


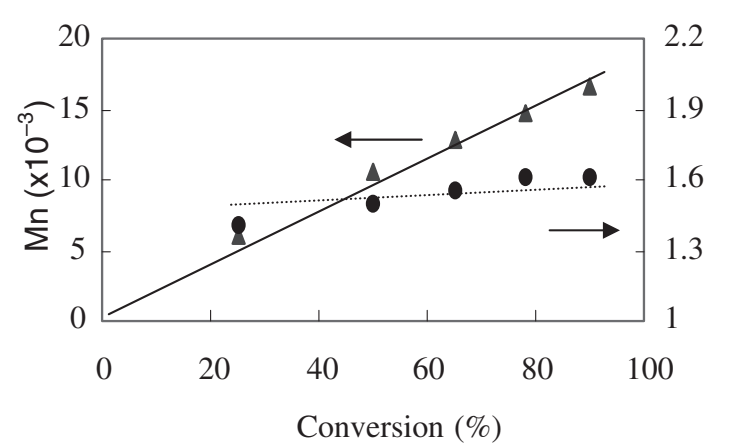

Figure 1. Conversion dependence of $M_{\mathrm{n}}(\mathbf{\Delta})$ and $M_{\mathrm{w}} / M_{\mathrm{n}}$ in the bulk ATRP using PMDETA as ligand at $110^{\circ} \mathrm{C}$.

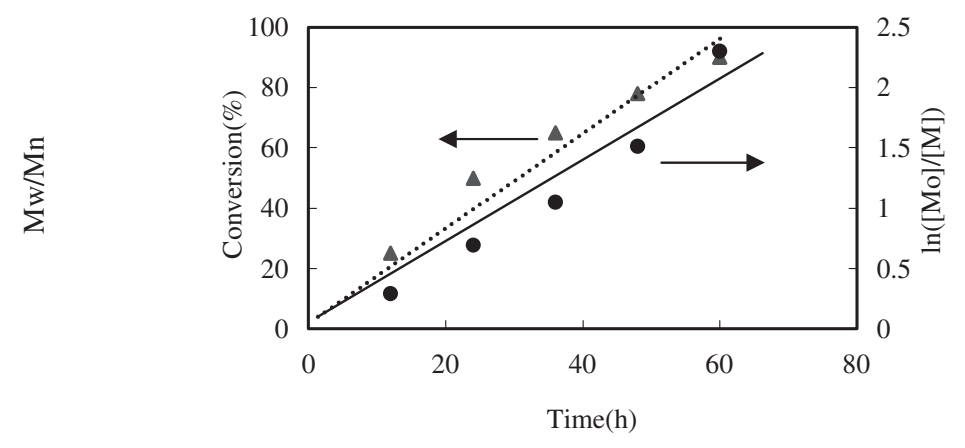

Figure 2. Polymerization time dependence of conversion $(\mathbf{\Delta})$ and $\ln \left(\left[M_{0}\right] /[M]\right)(\bullet)$ in the bulk ATRP using PMDETA as ligand at $110^{\circ} \mathrm{C}$.
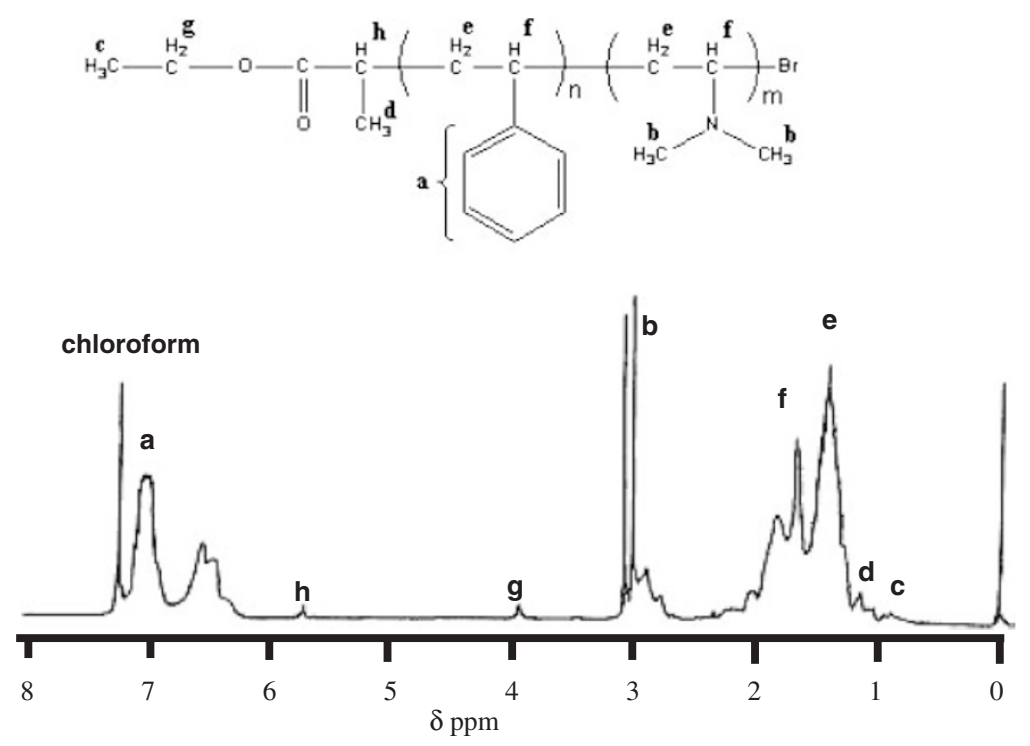

Figure 3. ${ }^{1} \mathrm{H}$ NMR spectrum of PSt- $b$-PDMAA.

concentration at time of $t$ ), against polymerization time gave straight lines passing through the origin zero, which showed constant concentrations of the propagating chains during the polymerizations. ${ }^{24}$ In addition, the polymerization rate (conversion increase ratio) beyond $40 \mathrm{~h}$ became slightly smaller than that at the initial stage and the polydispersities increased with increasing reaction time. These observations consist with Matyjaszewski's report. ${ }^{17}$ An increase in the copolymer molecular weights with the conversion increase may reduce chain mobility and propagation velocity, besides the concentration of free radicals became higher, which increased the probability of spontaneous termination reactions resulting in the polydispersity increase.

FT-IR experiments were carried out to characterize the PSt- $b$-PDMAA structure. We compared the representative FT-IR spectrum of PSt- $b$-PDMAA with that of PSt. The vibration bands characteristic of DMAA block assigned to the $\mathrm{C}=\mathrm{O}$ stretching are observed at around $1660 \mathrm{~cm}^{-1}$, confirming the presence of
DMAA block in the polymers. We investigated the block length of PSt- $b$-PDMAA by ${ }^{1} \mathrm{H}$ NMR. As shown in Figure 3, the integration ratio of DMAA protons to PSt protons, $b / a$, was estimated to be 1.3 that was in good agreement with the unit ratio of two blocks calculated based on the GPC results. As a consequence, we successfully made ATRP-syntheses of the amphiphilic block copolymers of PSt- $b$ PDMAA.

\section{Self-assembly of PSt-b-PDMAA}

Since PSt- $b$-PDMAA has an amphiphilic structure, they may self-assemble to form the nanoparticles in water. PSt- $b$-PDMAA was self-assembled by solvent exchange from DMF into the water to give the bluish translucent solution which showed a Tyndall phenomenon by laser irradiation $(635 \mathrm{~nm})$. This phenomenon suggests the formation of some self-assembled particles such as micelles and vesicles. We performed dynamic laser light scattering (DLLS) measurement of the solutions. The hydrodynamic radius, $R_{\mathrm{h}}$, of DLLS 


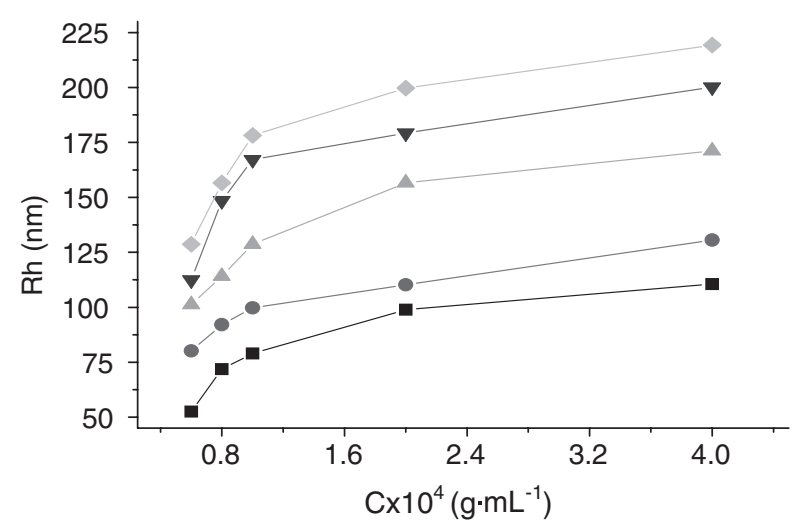

Figure 4. Change in hydrodynamic radius, $\mathrm{Rh}$, as a function of the copolymer concentration, P32 ( $\mathbf{\square})$, P77 (•), P101 ( $\mathbf{\Delta})$, P121 ( $)$, P139 ( ).

measurement showed no dependence on a scattering angle change from $6^{\circ}$ to $152^{\circ}$, in spite of a slight increase in a polydispersity index. We selected a typical scattering angle of $45^{\circ}$ for the following DLLS measurement. The DLLS diagram showed one peak with a single top, showing the polymer was self-assembled to form nanoparticles. Figure 4 shows the nanoparticle size change as a function of the concentration of the polymers. $R_{\mathrm{h}}$, of nanoparticles increased from 53 to $111 \mathrm{~nm}$ for P32, from 80 to $130 \mathrm{~nm}$ for $\mathrm{P} 77$, from 101 to $171 \mathrm{~nm}$ for P101, from 112 to $200 \mathrm{~nm}$ for P121, and from 129 to $219 \mathrm{~nm}$ for P139, when the polymer concentration increased from 0.06 to $0.4 \mathrm{mg} \mathrm{mL}^{-1}$. In the presence of $\mathrm{NaCl}$, the copolymers also showed $R_{\mathrm{h}}$ values in the analogous ranges (Figure 5). On the other hand, the lengths of PSt- $b$ PDMAA calculated under the assumption that the polymer backbones adopt an all-trans fully extended conformation were $14.3,25.6,31.6,36.6$, and 41.1 $\mathrm{nm}$, respectively (length of each blocks are summarized in Table I). These values were much smaller than the $R_{\mathrm{h}}$ of nanoparticles, indicating PSt- $b$-PDMAA

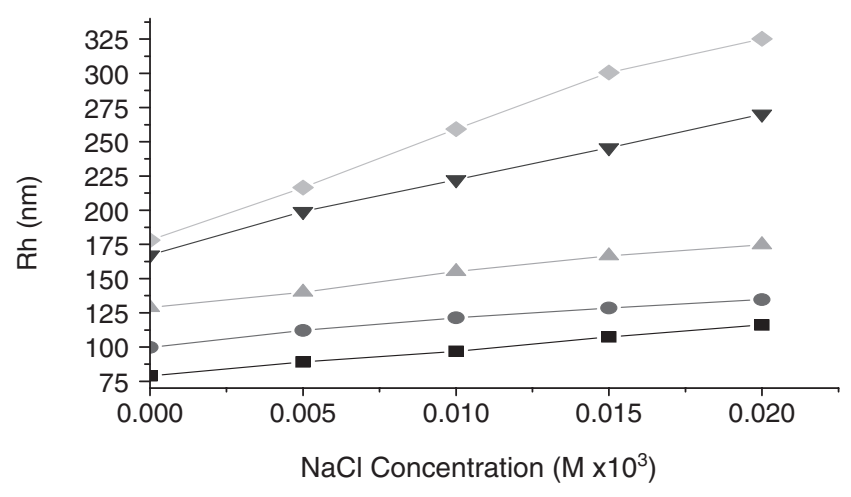

Figure 5. Change in hydrodynamic radius, $R_{\mathrm{h}}$, as a function

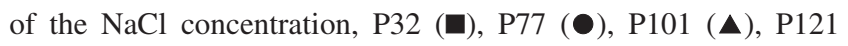
$(\boldsymbol{\nabla})$, P139 (•).
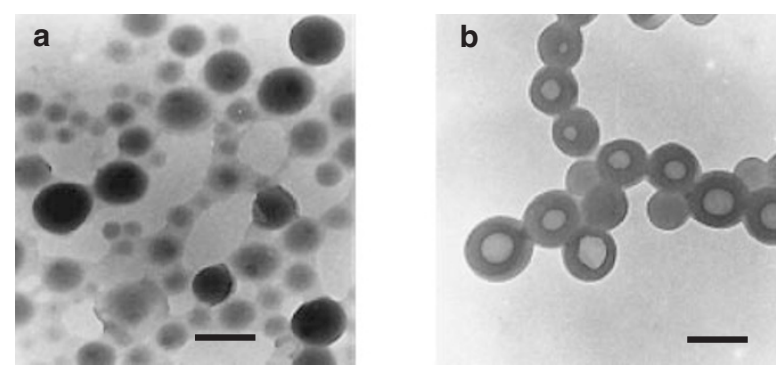

Figure 6. TEM images of the dried samples of PSt- $b$ PDMAA from the water-dispersion state at a concentration of $0.2 \mathrm{mg} \mathrm{mL}^{-1}$. (a) P32, (b) P121. Scale bars: $100 \mathrm{~nm}$.

did not form a simple micelle structure.

The morphology of the particles was investigated by transmission electron microscopy (TEM). In Figure 6, we show the TEM images of copolymers P32 and P121 and in both we can find spherical particles with a radius of and $15-40$ and $30-55 \mathrm{~nm}$, respectively. The size is smaller than $R_{\mathrm{h}}$, suggesting the particles are well hydrated in water. Around the center of the spherical matters for P121 sample, we can see a thinner region surrounded by a thicker region, and clearly confirm the boundary between the thinner and the thicker ones. This observation indicated that the PSt- $b$-PDMAA formed the vesicle particles with a hollow structure. Although the thickness of the thicker region in TEM is estimated to be about 15$30 \mathrm{~nm}$ which seems to be smaller than the copolymer chain length, the dry process to prepare the TEM sample may reduce the vesicular bilayer spacing. The thinner region of the P32 sample was not confirmed in Figure 6a, presumably due to too small hollow region.

We investigated the controllability of the vesicle size. Figure 4 shows the polymer concentration dependence of $R_{\mathrm{h}}$ in all the copolymers with a fixed length of hydrophobic PSt block. $R_{\mathrm{h}}$ increased with an increase in the polymer concentration but were much higher than the twice length of the copolymer chains with a fully-extended conformation; 29.2, 51.2, 63.2, 73.2, and $82.2 \mathrm{~nm}$ in P32, P77, P101, $\mathrm{P} 121$, and P139 respectively. Then the change in the vesicle size is not attributed to the copolymer conformation change leading to a bilayer thickness change, but to the size change in the hollow region. Since the vesicles generally keep in the equilibrated state with surrounding polymer solution, a higher association number in the vesicle bilayer was made in a higher polymer concentration. The higher association number simply increased the circumference length of the vesicle, forming the larger one. In addition, the large number of polymer chains in vesicular inner side having a higher curvature sometimes raised the vesicle size by the interchain volume-exclusion ef- 
fects, which may show an effect on the size increase to some extent. In any concentration, the block length of hydrophilic PDMAA strongly influenced the vesicle size. In this case, the interchain volume-exclusion effects of the longer hydrophilic segment in the bilayer inner side were more effective on the size increase rather than the association number. We investigated the effects of salt $\mathrm{NaCl}$ on the size of the copolymer vesicle. Figure 5 showed the ionic strength dependence of $R_{\mathrm{h}}$, where the polymer concentration value was fixed in $1 \times 10^{-5} \mathrm{~g} \mathrm{~mL}^{-1}$. An increase in $\mathrm{NaCl}$ concentration increased the vesicle size. An increase in the ionic strength increases the polarity of the aqueous milieu, increased the hydrophobic interaction of the PSt block. This effect can induce the increase in the association number and then vesicle size. Overall, the solution environment controlled the vesicle size.

\section{CONCLUSIONS}

We synthesized the PSt-Br macroinitiator by ATRP. The sequential polymerization successfully yielded amphiphilic block copolymers of PSt- $b$ PDMAA that were characterized by IR, ${ }^{1} \mathrm{H}$ NMR and GPC. Kinetic study suggested that the polymerization follows ATRP mechanism and the length of PDMAA block is controllable with a narrow distribution. Copolymers can self-assemble in water to form the vesicle whose size was dependent on the composition of diblock copolymers. The size of vesicles increased as the polymer and $\mathrm{NaCl}$ concentration increased. Thus the precisely synthesized PSt- $b$ PDMAA formed the size-controllable hollow matters by one-step process. If the bioactive molecules are contained in the hollow region, the dynamic nanobiomaterials may be developed.

Acknowledgment. This work was financially supported by Foundation for University Key Teacher by the Ministry of Education, P. R. China (2000-65), National Science Foundation of China 50443012 and Sekisui Integrated Research.

\section{REFERENCES}

1. J. C. M. Lee, H. Bermudez, B. M. Discher, M. A. Sheehan, Y. Y. Won, F. S. Bates, and D. E. Discher, Biotechnol.
Bioeng., 73, 135 (2001).

2. A. Graff, M. Sauer, P. D. Gelder, and W. Meier, Proc. Natl. Acad. Sci. U.S.A., 99, 5064 (2002).

3. V. S. Trubetsloy, M. D. Frank-Kanebetsky, and K. R. Whiteman, Acad. Radiol., 3, 232 (1996).

4. R. Nagarajan, M. Barry, and E. Ruckenstein, Langmuir, 2, 210 (1986).

5. X. Y. Liu, M. Jiang, S. L. Yang, M. Q. Chen, C. Yang, and K. Wu, Angew. Chem., Int. Ed., 41, 2950 (2002).

6. Z. Niu, Z. Yang, Z. Hu, Y. Lu, and C. C. Han, Adv. Funct. Mater., 13, 949 (2003).

7. T. Sanji, Y. Nakatsuka, S. Ohnishi, and H. Sakurai, Macromolecules, 33, 8524 (2000).

8. P. L. Soo and A. Eisenberg, J. Polym. Sci., Part B: Polym. Phys., 42, 923 (2004).

9. M. Antonietti and S. Förster, Adv. Mater., 15, 1323 (2003).

10. H. S. Peng, D. Y. Chen, and M. Jiang, Langmuir, 19, 10989 (2003).

11. D. Neugebauer and K. Matyjaszewski, Macromolecules, 36, 2598 (2003).

12. J. N. Kizhakkedathu and D. E. Brooks, Macromolecules, 36, 591 (2003).

13. J. Z. Du and Y. M. Chen, Macromolecules, 37, 5710 (2004).

14. J. S. Wang and K. Matyjaszewski, J. Am. Chem. Soc., 117, 5614 (1995).

15. K. L. Robinson, M. V. de Paz-Báñez, X. S. Wang, and S. P. Armes, Macromolecules, 34, 5799 (2001).

16. J. N. Kizhakkedathu, R. Norris-Jones, and D. E. Brooks, Macromolecules, 37, 734 (2004).

17. M. Teodorescu and K. Matyjaszewski, Macromolecules, 32, 4826 (1999).

18. T. S. C. Pai, C. B. Kowollik, T. P. Davis, and M. H. Stenzel, Polymer, 45, 4383 (2004).

19. J. F. Lutz, D. Neugebauer, and K. Matyjaszewski, J. Am. Chem. Soc., 125, 6986 (2003).

20. J. S. Wang and K. Matyjaszewski, Macromolecules, 28, 7901 (1995).

21. a) J. Xia and K. Matyjaszewski, Macromolecules, 30, 7697 (1997).

b) J. T. Rademacher, M. Baun, M. E. Pallack, and W. J. Brittain, Macromolecules, 33, 284 (2000).

22. a) H. Zhu, S. Y. Liu, and M. Jiang, Chem. J. Chin. Univ., 23, 138 (2002).

b) H. B. Sonmez, B. F. Semkal, D. C. Sherrington, and N. Bicak, React. Funct. Polym., 55, 1 (2003).

23. J. N. Kizhakkedathu, K. R. Kumar, D. Goodman, and D. E. Brooks, Polymer in press.

24. a) L. F. Zhang and A. Esenberg, Science, 268, 1728 (1995). b) L. F. Zhang and A. Esenberg, J. Am. Chem. Soc., 118, 3168 (1996). 